\title{
Oscillatory behavior of third-order neutral delay differential equations with distributed deviating arguments
}

Yibing Sun ${ }^{1 *}$ and Yige Zhao ${ }^{1}$

\section{"Correspondence:}

sun_yibing@126.com

'School of Mathematical Sciences,

University of Jinan, Jinan, P.R. China

\begin{abstract}
The main contribution of this paper is to establish some new criteria, which ensure that every solution of third-order neutral delay differential equations with distributed deviating arguments is either oscillatory or tends to zero. The obtained theorems extend and improve several known results in the literature. Two examples are provided to illustrate the main results.
\end{abstract}

MSC: $34 \mathrm{~K} 11$

Keywords: Oscillation; Asymptotic property; Distributed deviating arguments; Riccati transformation

\section{Introduction}

Over the last several years, an increasing attention has been given to the oscillation theory and asymptotic behavior of various classes of second-order and high-order differential equations and dynamic equations on time scales [1-17]. So far, much research activity concerns the oscillation problem of the third-order (TO) neutral differential and dynamic equations [18-26]. Recently, the research focus has been shifted to the study of the TO differential equations (DE) with distributed deviating arguments (DDA), and some results can be found in [27-35].

Li et al. [3] investigated

$$
\left(r(t)\left|z^{\prime}(t)\right|^{\alpha-1} z^{\prime}(t)\right)^{\prime}+\int_{a}^{b} q(t, \xi)|x[g(t, \xi)]|^{\alpha-1} x[g(t, \xi)] d \sigma(\xi)=0,
$$

where $z(t)=x(t)+p(t) x(\tau(t)), 0 \leq p(t) \leq p_{0}<\infty$ and $\alpha \geq 1$ is a constant. Under the methods proposed by $\mathrm{Li}$ et al. [3, 20], Jiang and Li [30] studied the following equation with DDA:

$$
\left(r(t)\left|z^{\prime \prime}(t)\right|^{\alpha-1} z^{\prime \prime}(t)\right)^{\prime}+\int_{a}^{b} q(t, \xi)|x[g(t, \xi)]|^{\alpha-1} x[g(t, \xi)] d \sigma(\xi)=0,
$$

where $\alpha>0$ is a constant, and obtained several theorems for (1.1) whenever

$$
\int_{t_{0}}^{\infty} r^{-\frac{1}{\alpha}}(t) d t=\infty, \quad \text { or } \quad \int_{t_{0}}^{\infty} r^{-\frac{1}{\alpha}}(t) d t<\infty
$$

(c) The Author(s) 2019. This article is distributed under the terms of the Creative Commons Attribution 4.0 International License (http://creativecommons.org/licenses/by/4.0/), which permits unrestricted use, distribution, and reproduction in any medium, provided you give appropriate credit to the original author(s) and the source, provide a link to the Creative Commons license, and indicate if changes were made. 
Furthermore, Elabbasy and Moaaz [31], and Wang et al. [32] examined a TODE with DDA under the assumption $0 \leq p(t) \leq P<1$. However, the obtained oscillation theorems cannot be applied when $p(t) \geq 1$. Then Tunç [33] utilized a new technique, different from the existing methods, to give some criteria for a TODE with DDA, when $p(t) \geq 1$.

The main objective here is to establish several oscillation criteria for the TO neutral delay (ND) DE with DDA

$$
E_{2}^{\prime}(t)+\int_{a}^{b} q(t, \xi)|x(g(t, \xi))|^{\alpha_{3}-1} x(g(t, \xi)) d \sigma(\xi)=0
$$

where $t \geq t_{0}>0$,

$$
\begin{aligned}
& E_{2}(t)=r_{2}(t)\left|E_{1}^{\prime}(t)\right|^{\alpha_{2}-1} E_{1}^{\prime}(t), \\
& E_{1}(t)=r_{1}(t)\left|z^{\prime}(t)\right|^{\alpha_{1}-1} z^{\prime}(t), \\
& z(t)=x(t)+p(t) x(\tau(t)),
\end{aligned}
$$

and $\alpha_{1}, \alpha_{2}$, and $\alpha_{3}$ are positive constants. We assume that:

(A1) $r_{i}(t) \in C\left(\left[t_{0}, \infty\right),(0, \infty)\right), \int_{t_{0}}^{\infty} r_{i}^{-\frac{1}{\alpha_{i}}}(t) d t=\infty, i=1,2$;

(A2) $p(t) \in C\left(\left[t_{0}, \infty\right),[1, \infty)\right)$ with $p(t) \not \equiv 1$, and $q(t, \xi) \in C\left(\left[t_{0}, \infty\right) \times[a, b],[0, \infty)\right)$ with $q(t, \xi) \not \equiv 0$ eventually;

(A3) $\tau(t) \in C^{1}\left(\left[t_{0}, \infty\right), \mathbb{R}\right), \tau(t) \leq t, \tau^{\prime}(t)>0$ and $\lim _{t \rightarrow \infty} \tau(t)=\infty$;

(A4) $g(t, \xi) \in C\left(\left[t_{0}, \infty\right) \times[a, b], \mathbb{R}\right)$ is a nondecreasing function for $\xi$, which satisfies $\liminf _{t \rightarrow \infty} g(t, \xi)=\infty$ for $\xi \in[a, b]$;

(A5) $\sigma(\xi) \in C([a, b], \mathbb{R})$ is nondecreasing and the integral of (1.2) is taken in the Riemann-Stieltjies sense.

This article is organized in the following manner. Section 2 presents three lemmas to prove our results. Section 3 establishes some new oscillation criteria for (1.2). Two examples finalize this article.

\section{Some lemmas}

For simplicity, we use some notations for sufficiently large $t_{1}$ with $t_{1} \geq t_{0}$ as below:

$$
\begin{aligned}
& g_{1}(t)=g(t, a), \quad g_{2}(t)=g(t, b), \quad d_{+}(t)=\max \{0, d(t)\}, \\
& \delta_{1}\left(t, t_{1}\right)=\int_{t_{1}}^{t}\left(\frac{1}{r_{2}(s)}\right)^{\frac{1}{\alpha_{2}}} d s, \quad \delta_{2}\left(t, t_{1}\right)=\left(\frac{\delta_{1}\left(t, t_{1}\right)}{r_{1}(t)}\right)^{\frac{1}{\alpha_{1}}}, \\
& \delta_{3}\left(t, t_{1}\right)=\int_{t_{1}}^{t} \delta_{2}\left(s, t_{1}\right) d s, \quad t \geq t_{1} .
\end{aligned}
$$

Furthermore, assume that

$$
\begin{aligned}
& p_{1}(t)=\frac{1}{p\left(\tau^{-1}(t)\right)}\left(1-\frac{1}{p\left(\tau^{-1}\left(\tau^{-1}(t)\right)\right)}\right)>0, \\
& p_{2}(t)=\frac{1}{p\left(\tau^{-1}(t)\right)}\left(1-\frac{\delta_{3}\left(\tau^{-1}\left(\tau^{-1}(t)\right), t_{1}\right)}{p\left(\tau^{-1}\left(\tau^{-1}(t)\right)\right) \delta_{3}\left(\tau^{-1}(t), t_{1}\right)}\right)>0,
\end{aligned}
$$




$$
\begin{aligned}
& q_{1}(t)=\int_{a}^{b} q(t, \xi) p_{1}^{\alpha_{3}}(g(t, \xi)) d \sigma(\xi), \\
& q_{2}(t)=\int_{a}^{b} q(t, \xi) p_{2}^{\alpha_{3}}(g(t, \xi)) d \sigma(\xi) .
\end{aligned}
$$

Lemma 2.1 Assume that (A1)-(A5) hold. Furthermore, let $x(t)$ be an eventually positive solution of (1.2). Then $z(t)$ satisfies either

$$
\text { (I) } \quad z(t)>0, \quad z^{\prime}(t)>0, \quad E_{1}^{\prime}(t)>0, \quad E_{2}^{\prime}(t) \leq 0,
$$

or

$$
\text { (II) } \quad z(t)>0, \quad z^{\prime}(t)<0, \quad E_{1}^{\prime}(t)>0, \quad E_{2}^{\prime}(t) \leq 0 .
$$

Proof From the condition of Lemma 2.1, there exists a $t_{1} \geq t_{0}$ such that

$$
x(t)>0, \quad x(\tau(t))>0 \quad \text { and } \quad x(g(t, \xi))>0, \quad \xi \in[a, b],
$$

for $t \geq t_{1}$. Then (1.2) implies that

$$
E_{2}^{\prime}(t)=-\int_{a}^{b} q(t, \xi)|x(g(t, \xi))|^{\alpha_{3}-1} x(g(t, \xi)) d \sigma(\xi) \leq 0
$$

which means that $E_{2}(t)$ is nonincreasing and of constant sign, and $E_{1}^{\prime}(t)$ is also of constant sign. We claim that $E_{1}^{\prime}(t)>0$. To prove this, assume on the contrary that $E_{1}^{\prime}(t)<0$ and there exists $M_{1}$, s.t. for $t \geq t_{2} \geq t_{1}$,

$$
E_{2}(t) \leq-M_{1}<0
$$

Then

$$
\left|E_{1}^{\prime}(t)\right| \geq\left(\frac{M_{1}}{r_{2}(t)}\right)^{\frac{1}{\alpha_{2}}}>0 .
$$

We integrate (2.5) to get

$$
E_{1}(t) \leq E_{1}\left(t_{2}\right)-M_{1}^{\frac{1}{\alpha_{2}}} \int_{t_{2}}^{t}\left(\frac{1}{r_{2}(s)}\right)^{\frac{1}{\alpha_{2}}} d s .
$$

Letting $t \rightarrow \infty$ and from (A1), we obtain $\lim _{t \rightarrow \infty} E_{1}(t)=-\infty$. Then there exist constants $M_{2}$ and $t_{3} \geq t_{2}$ such that

$$
E_{1}(t) \leq-M_{2}<0, \quad t \geq t_{3}
$$

which yields that

$$
\left|z^{\prime}(t)\right| \geq\left(\frac{M_{2}}{r_{1}(t)}\right)^{\frac{1}{\alpha_{1}}}>0 .
$$


Integrating the latter inequality from $t_{3}$ to $t$, we have

$$
z(t) \leq z\left(t_{3}\right)-M_{2}^{\frac{1}{\alpha_{1}}} \int_{t_{3}}^{t}\left(\frac{1}{r_{1}(s)}\right)^{\frac{1}{\alpha_{1}}} d s
$$

We use (A1) again to have $\lim _{t \rightarrow \infty} z(t)=-\infty$, which contradicts $z(t)>0$. Hence, $E_{1}^{\prime}(t)>0$ for $t \geq t_{1}$, and $z(t)$ has properties (I) and (II).

Lemma 2.2 Assume that (A1)-(A5) and (2.1) hold. Furthermore, suppose that $x(t)$ is an eventually positive solution of (1.2) and $z(t)$ has property (II) of Lemma 2.1. If

$$
\int_{t_{0}}^{\infty}\left[\frac{1}{r_{1}(u)} \int_{u}^{\infty}\left(\frac{1}{r_{2}(v)} \int_{v}^{\infty} q_{1}(s) d s\right)^{\frac{1}{\alpha_{2}}} d v\right]^{\frac{1}{\alpha_{1}}} d u=\infty,
$$

then $\lim _{t \rightarrow \infty} x(t)=0$.

Proof One can see that (1.3) yields (see [33])

$$
z\left(\tau^{-1}(t)\right)=x\left(\tau^{-1}(t)\right)+p\left(\tau^{-1}(t)\right) x(t)
$$

which can be rewritten as

$$
\begin{aligned}
x(t) & \geq \frac{z\left(\tau^{-1}(t)\right)}{p\left(\tau^{-1}(t)\right)}-\frac{z\left(\tau^{-1}\left(\tau^{-1}(t)\right)\right)}{p\left(\tau^{-1}(t)\right) p\left(\tau^{-1}\left(\tau^{-1}(t)\right)\right)} \\
& \geq p_{1}(t) z\left(\tau^{-1}(t)\right) .
\end{aligned}
$$

Combining (2.4) and (2.8), we get

$$
\begin{aligned}
E_{2}^{\prime}(t) & \leq-\int_{a}^{b} q(t, \xi) p_{1}^{\alpha_{3}}(g(t, \xi)) z^{\alpha_{3}}\left(\tau^{-1}(g(t, \xi))\right) d \sigma(\xi) \\
& \leq-q_{1}(t) z^{\alpha_{3}}\left(\tau^{-1}\left(g_{2}(t)\right)\right)
\end{aligned}
$$

based on the fact that $z^{\prime}(t)<0$ for $t \geq t_{1}$. Since $z(t)$ has property (II) of Lemma 2.1, one gets $\lim _{t \rightarrow \infty} z(t)=l \geq 0$. We claim that $l=0$. Indeed, if we assume on the contrary that $l>0$, then there exists $t_{2} \geq t_{1}$ s.t. $\tau^{-1}\left(g_{2}(t)\right) \geq t_{2}$ and $z\left(\tau^{-1}\left(g_{2}(t)\right)\right) \geq l, t \geq t_{2}$. Inequality (2.9) then yields

$$
E_{2}^{\prime}(t) \leq-l^{\alpha_{3}} q_{1}(t)
$$

We integrate (2.10) to get

$$
r_{2}(t)\left(E_{1}^{\prime}(t)\right)^{\alpha_{2}} \geq l^{\alpha_{3}} \int_{t}^{\infty} q_{1}(s) d s
$$

which indicates that

$$
E_{1}^{\prime}(t) \geq\left(\frac{l^{\alpha_{3}}}{r_{2}(t)} \int_{t}^{\infty} q_{1}(s) d s\right)^{\frac{1}{\alpha_{2}}}
$$


Integrating (2.11) from $t$ to $\infty$, we have

$$
-E_{1}(t) \geq l^{\frac{\alpha_{3}}{\alpha_{2}}} \int_{t}^{\infty}\left(\frac{1}{r_{2}(v)} \int_{v}^{\infty} q_{1}(s) d s\right)^{\frac{1}{\alpha_{2}}} d v
$$

and then

$$
\left|z^{\prime}(t)\right| \geq l^{\frac{\alpha_{3}}{\alpha_{1} \alpha_{2}}}\left[\frac{1}{r_{1}(t)} \int_{t}^{\infty}\left(\frac{1}{r_{2}(v)} \int_{v}^{\infty} q_{1}(s) d s\right)^{\frac{1}{\alpha_{2}}} d v\right]^{\frac{1}{\alpha_{1}}}
$$

since $z^{\prime}(t)<0$ for $t \geq t_{2}$. Integrating the latter inequality from $t_{2}$ to $\infty$, we get

$$
z\left(t_{2}\right)>l^{\frac{\alpha_{3}}{\alpha_{1} \alpha_{2}}} \int_{t_{2}}^{\infty}\left[\frac{1}{r_{1}(u)} \int_{u}^{\infty}\left(\frac{1}{r_{2}(v)} \int_{v}^{\infty} q_{1}(s) d s\right)^{\frac{1}{\alpha_{2}}} d v\right]^{\frac{1}{\alpha_{1}}} d u
$$

which contradicts (2.6). Thus, we obtain $l=0$ and $\lim _{t \rightarrow \infty} x(t)=0$, since $0<x(t) \leq z(t)$.

Lemma 2.3 Assume that (A1)-(A5) and (2.2) hold. Furthermore, suppose that $x(t)$ is an eventually positive solution of (1.2) and $z(t)$ has property (I) of Lemma 2.1. Then for $t \geq$ $t_{1} \geq t_{0}$

$$
\left[r_{2}(t)\left(\left(r_{1}(t)\left(z^{\prime}(t)\right)^{\alpha_{1}}\right)^{\prime}\right)^{\alpha_{2}}\right]^{\prime}+q_{2}(t) z^{\alpha_{3}}\left(\tau^{-1}\left(g_{1}(t)\right)\right) \leq 0
$$

Proof Property (I) of $z(t)$ yields

$$
E_{2}(t)=r_{2}(t)\left(\left(r_{1}(t)\left(z^{\prime}(t)\right)^{\alpha_{1}}\right)^{\prime}\right)^{\alpha_{2}}>0
$$

Applying the monotonicity of $E_{2}^{\frac{1}{\alpha_{2}}}(t)$ gives

$$
\begin{aligned}
r_{1}(t)\left(z^{\prime}(t)\right)^{\alpha_{1}} & =r_{1}\left(t_{1}\right)\left(z^{\prime}\left(t_{1}\right)\right)^{\alpha_{1}}+\int_{t_{1}}^{t} \frac{r_{2}^{\frac{1}{\alpha_{2}}}(s)\left(r_{1}(s)\left(z^{\prime}(s)\right)^{\alpha_{1}}\right)^{\prime}}{r_{2}^{\frac{1}{\alpha_{2}}}(s)} d s \\
& \geq \delta_{1}\left(t, t_{1}\right) r_{2}^{\frac{1}{\alpha_{2}}}(t)\left(r_{1}(t)\left(z^{\prime}(t)\right)^{\alpha_{1}}\right)^{\prime} .
\end{aligned}
$$

It can be seen from (2.13) that

$$
\left(\frac{r_{1}(t)\left(z^{\prime}(t)\right)^{\alpha_{1}}}{\delta_{1}\left(t, t_{1}\right)}\right)^{\prime} \leq 0
$$

which, together with $r_{1}(t)\left(z^{\prime}(t)\right)^{\alpha_{1}}>0$, yields that $z^{\prime}(t) / \delta_{2}\left(t, t_{1}\right)$ is nonincreasing for $t \geq t_{1}$. Therefore,

$$
z(t)=z\left(t_{1}\right)+\int_{t_{1}}^{t} \frac{z^{\prime}(s)}{\delta_{2}\left(s, t_{1}\right)} \delta_{2}\left(s, t_{1}\right) d s \geq \frac{\delta_{3}\left(t, t_{1}\right)}{\delta_{2}\left(t, t_{1}\right)} z^{\prime}(t)
$$

which leads to

$$
\left(\frac{z(t)}{\delta_{3}\left(t, t_{1}\right)}\right)^{\prime} \leq 0
$$


Then for $t \geq t_{2} \geq t_{1}$,

$$
z\left(\tau^{-1}\left(\tau^{-1}(t)\right)\right) \leq \frac{\delta_{3}\left(\tau^{-1}\left(\tau^{-1}(t)\right), t_{1}\right) z\left(\tau^{-1}(t)\right)}{\delta_{3}\left(\tau^{-1}(t), t_{1}\right)},
$$

due to $\tau^{-1}(t) \leq \tau^{-1}\left(\tau^{-1}(t)\right)$. Substituting (2.16) into (2.7), one has

$$
x(t) \geq p_{2}(t) z\left(\tau^{-1}(t)\right)
$$

which leads to

$$
x(g(t, \xi)) \geq p_{2}(g(t, \xi)) z\left(\tau^{-1}\left(g_{1}(t)\right)\right) .
$$

Combining (1.2) and (2.17), we obtain (2.12).

\section{Main results}

We respectively consider two cases $g_{1}(t) \leq \tau(t)$ and $g_{1}(t) \geq \tau(t)$ for $t \geq t_{0}$. Now, we begin with the first case.

Theorem 3.1 Assume that (A1)-(A5), (2.1), (2.2), and (2.6) hold, and $g_{1}(t) \leq \tau(t)$. If there exists $\rho(t) \in C^{1}\left(\left[t_{0}, \infty\right),(0, \infty)\right)$ s.t.

$$
\begin{gathered}
\limsup _{t \rightarrow \infty} \int_{t_{*}}^{t}\left[\rho(s) q_{2}(s)\left(\frac{\delta_{3}\left(\tau^{-1}\left(g_{1}(s)\right), t_{1}\right)}{\delta_{3}\left(s, t_{1}\right)}\right)^{\alpha_{3}}\right. \\
\left.-\frac{\lambda\left(\rho_{+}^{\prime}(s)\right)^{\alpha_{1} \alpha_{2}+1}}{\left(\rho(s) \gamma(s) \delta_{2}\left(s, t_{1}\right)\right)^{\alpha_{1} \alpha_{2}}}\right] d s=\infty,
\end{gathered}
$$

for $t_{1}$ and $t_{*}$ with $t_{*} \geq t_{1} \geq t_{0}$, where

$$
\begin{aligned}
& \lambda=\left(\frac{\alpha_{1} \alpha_{2}}{\alpha_{3}}\right)^{\alpha_{1} \alpha_{2}}\left(\frac{1}{\alpha_{1} \alpha_{2}+1}\right)^{\alpha_{1} \alpha_{2}+1}, \\
& \gamma(t)= \begin{cases}m_{1}\left(\delta_{3}\left(t, t_{1}\right)\right)^{\frac{\alpha_{3}}{\alpha_{1} \alpha_{2}}-1}, \quad m_{1} \text { is any positive constant, } & \text { if } \alpha_{1} \alpha_{2}>\alpha_{3}, \\
m_{2}, \quad m_{2} \text { is any positive constant, } & \text { if } \alpha_{1} \alpha_{2} \leq \alpha_{3},\end{cases}
\end{aligned}
$$

then every solution of (1.2) is either oscillatory or tends to zero.

Proof Suppose that (1.2) has a nonoscillatory solution $x(t)$. We may assume that (2.3) holds for $t \geq t_{1} \geq t_{0}$. So we have that $z(t)$ is positive and satisfies the two properties for $t \geq t_{1}$.

We first consider property (I). Define $\omega(t)$ by

$$
\omega(t)=\rho(t) \frac{r_{2}(t)\left(\left(r_{1}(t)\left(z^{\prime}(t)\right)^{\alpha_{1}}\right)^{\prime}\right)^{\alpha_{2}}}{z^{\alpha_{3}}(t)}, \quad t \geq t_{1}
$$

Then $\omega(t)>0$ and

$$
\begin{aligned}
\omega^{\prime}(t)= & \rho^{\prime}(t) \frac{r_{2}(t)\left(\left(r_{1}(t)\left(z^{\prime}(t)\right)^{\alpha_{1}}\right)^{\prime}\right)^{\alpha_{2}}}{z^{\alpha_{3}}(t)}+\rho(t)\left[\frac{\left[r_{2}(t)\left(\left(r_{1}(t)\left(z^{\prime}(t)\right)^{\alpha_{1}}\right)^{\prime}\right)^{\alpha_{2}}\right]^{\prime}}{z^{\alpha_{3}}(t)}\right. \\
& \left.-\frac{\alpha_{3} r_{2}(t)\left(\left(r_{1}(t)\left(z^{\prime}(t)\right)^{\alpha_{1}}\right)^{\prime}\right)^{\alpha_{2}} z^{\prime}(t)}{z^{\alpha_{3}+1}(t)}\right]
\end{aligned}
$$




$$
\begin{gathered}
=\frac{\rho^{\prime}(t)}{\rho(t)} \omega(t)+\rho(t) \frac{\left[r_{2}(t)\left(\left(r_{1}(t)\left(z^{\prime}(t)\right)^{\alpha_{1}}\right)^{\prime}\right)^{\alpha_{2}}\right]^{\prime}}{z^{\alpha_{3}}(t)} \\
-\alpha_{3} \rho(t) \frac{r_{2}(t)\left(\left(r_{1}(t)\left(z^{\prime}(t)\right)^{\alpha_{1}}\right)^{\prime}\right)^{\alpha_{2}} z^{\prime}(t)}{z^{\alpha_{3}+1}(t)} .
\end{gathered}
$$

Based on (2.12), we have

$$
\frac{\left[r_{2}(t)\left(\left(r_{1}(t)\left(z^{\prime}(t)\right)^{\alpha_{1}}\right)^{\prime}\right)^{\alpha_{2}}\right]^{\prime}}{z^{\alpha_{3}}(t)} \leq-q_{2}(t)\left(\frac{z\left(\tau^{-1}\left(g_{1}(t)\right)\right)}{z(t)}\right)^{\alpha_{3}} .
$$

Since $g_{1}(t) \leq \tau(t)$, we get $\tau^{-1}\left(g_{1}(t)\right) \leq t$. Applying (2.15), we obtain

$$
\frac{z\left(\tau^{-1}\left(g_{1}(t)\right)\right)}{z(t)} \geq \frac{\delta_{3}\left(\tau^{-1}\left(g_{1}(t)\right), t_{1}\right)}{\delta_{3}\left(t, t_{1}\right)} .
$$

From (2.13), we have

$$
z^{\prime}(t) \geq \delta_{2}\left(t, t_{1}\right)\left(r_{2}(t)\left(\left(r_{1}(t)\left(z^{\prime}(t)\right)^{\alpha_{1}}\right)^{\prime}\right)^{\alpha_{2}}\right)^{\frac{1}{\alpha_{1} \alpha_{2}}} .
$$

We combine (3.4)-(3.7) to conclude that

$$
\begin{aligned}
\omega^{\prime}(t) \leq & \frac{\rho_{+}^{\prime}(t)}{\rho(t)} \omega(t)-\rho(t) q_{2}(t)\left(\frac{\delta_{3}\left(\tau^{-1}\left(g_{1}(t)\right), t_{1}\right)}{\delta_{3}\left(t, t_{1}\right)}\right)^{\alpha_{3}} \\
& -\frac{\alpha_{3} \delta_{2}\left(t, t_{1}\right)}{\rho^{\frac{1}{\alpha_{1} \alpha_{2}}}(t)} z^{\frac{\alpha_{3}}{\alpha_{1} \alpha_{2}}-1}(t) \omega^{\frac{1}{\alpha_{1} \alpha_{2}}+1}(t) .
\end{aligned}
$$

Next, we will compute $z^{\frac{\alpha_{3}}{\alpha_{1} \alpha_{2}}-1}(t)$ and consider the following two cases:

Case (i). Assume that $\alpha_{1} \alpha_{2}>\alpha_{3}$. Since $z(t) / \delta_{3}\left(t, t_{1}\right)$ is nonincreasing, due to (2.15), there exist constants $h_{1}>0$ and $t_{2} \geq t_{1}$ such that

$$
\frac{z(t)}{\delta_{3}\left(t, t_{1}\right)} \leq \frac{z\left(t_{2}\right)}{\delta_{3}\left(t_{2}, t_{1}\right)}=h_{1}, \quad t \geq t_{2}
$$

and

$$
z^{\frac{\alpha_{3}}{\alpha_{1} \alpha_{2}}-1}(t) \geq m_{1}\left(\delta_{3}\left(t, t_{1}\right)\right)^{\frac{\alpha_{3}}{\alpha_{1} \alpha_{2}}-1}
$$

where $m_{1}=h_{1}^{\frac{\alpha_{3}}{\alpha_{1} \alpha_{2}}-1}$.

Case (ii). Assume that $\alpha_{1} \alpha_{2} \leq \alpha_{3}$. Since $z^{\prime}(t)>0$, there exists $h_{2}>0$ such that

$$
z(t) \geq z\left(t_{1}\right)=h_{2}, \quad t \geq t_{1},
$$

and

$$
z^{\frac{\alpha_{3}}{\alpha_{1} \alpha_{2}}-1}(t) \geq m_{2}, \quad t \geq t_{1}
$$


where $m_{2}=h_{2}^{\frac{\alpha_{3}}{\alpha_{1} \alpha_{2}}-1}$. We combine (3.8) with (3.9) and (3.10) to have

$$
\begin{aligned}
\omega^{\prime}(t) \leq & \frac{\rho_{+}^{\prime}(t)}{\rho(t)} \omega(t)-\rho(t) q_{2}(t)\left(\frac{\delta_{3}\left(\tau^{-1}\left(g_{1}(t)\right), t_{1}\right)}{\delta_{3}\left(t, t_{1}\right)}\right)^{\alpha_{3}} \\
& -\frac{\alpha_{3} \gamma(t) \delta_{2}\left(t, t_{1}\right)}{\rho^{\frac{1}{\alpha_{1} \alpha_{2}}}(t)} \omega^{\frac{1}{\alpha_{1} \alpha_{2}}+1}(t) .
\end{aligned}
$$

By using the inequality (see [18])

$$
B u-A u^{\frac{1}{\alpha}+1} \leq \frac{\alpha^{\alpha}}{(\alpha+1)^{\alpha+1}} \frac{B^{\alpha+1}}{A^{\alpha}}, \quad A>0
$$

where

$$
B=\frac{\rho_{+}^{\prime}(t)}{\rho(t)}, \quad A=\frac{\alpha_{3} \gamma(t) \delta_{2}\left(t, t_{1}\right)}{\rho^{\frac{1}{\alpha_{1} \alpha_{2}}}(t)}, \quad \alpha=\alpha_{1} \alpha_{2}, u=\omega(t),
$$

we obtain

$$
\begin{aligned}
& \frac{\rho_{+}^{\prime}(t)}{\rho(t)} \omega(t)-\frac{\alpha_{3} \gamma(t) \delta_{2}\left(t, t_{1}\right)}{\rho^{\frac{1}{\alpha_{1} \alpha_{2}}}(t)} \omega^{\frac{1}{\alpha_{1} \alpha_{2}}}+1 \\
& \quad \leq\left(\frac{\alpha_{1} \alpha_{2}}{\alpha_{3} \rho(t) \gamma(t) \delta_{2}\left(t, t_{1}\right)}\right)^{\alpha_{1} \alpha_{2}}\left(\frac{\rho_{+}^{\prime}(t)}{\alpha_{1} \alpha_{2}+1}\right)^{\alpha_{1} \alpha_{2}+1} \\
& \quad=\frac{\lambda\left(\rho_{+}^{\prime}(t)\right)^{\alpha_{1} \alpha_{2}+1}}{\left(\rho(t) \gamma(t) \delta_{2}\left(t, t_{1}\right)\right)^{\alpha_{1} \alpha_{2}}} .
\end{aligned}
$$

We combine (3.11) and (3.13) to conclude that

$$
\omega^{\prime}(t) \leq-\rho(t) q_{2}(t)\left(\frac{\delta_{3}\left(\tau^{-1}\left(g_{1}(t)\right), t_{1}\right)}{\delta_{3}\left(t, t_{1}\right)}\right)^{\alpha_{3}}+\frac{\lambda\left(\rho_{+}^{\prime}(t)\right)^{\alpha_{1} \alpha_{2}+1}}{\left(\rho(t) \gamma(t) \delta_{2}\left(t, t_{1}\right)\right)^{\alpha_{1} \alpha_{2}}}
$$

We integrate the latter inequality to make

$$
\begin{aligned}
& \int_{t_{2}}^{t}\left[\rho(s) q_{2}(s)\left(\frac{\delta_{3}\left(\tau^{-1}\left(g_{1}(s)\right), t_{1}\right)}{\delta_{3}\left(s, t_{1}\right)}\right)^{\alpha_{3}}-\frac{\lambda\left(\rho_{+}^{\prime}(s)\right)^{\alpha_{1} \alpha_{2}+1}}{\left(\rho(s) \gamma(s) \delta_{2}\left(s, t_{1}\right)\right)^{\alpha_{1} \alpha_{2}}}\right] d s \\
& \quad \leq \omega\left(t_{2}\right)-\omega(t)<\omega\left(t_{2}\right)
\end{aligned}
$$

which contradicts (3.1).

Secondly, we investigate property (II) and deduce $\lim _{t \rightarrow \infty} x(t)=0$.

Theorem 3.2 Assume that (A1)-(A5), (2.1), (2.2), and (2.6) hold. Furthermore, suppose that $g_{1}(t) \leq \tau(t)$ and $\alpha_{1} \alpha_{2}=\alpha_{3}$. If there exists $\rho(t)$ s.t.

$$
\limsup _{t \rightarrow \infty} \int_{t_{*}}^{t}\left[\rho(s) q_{2}(s)\left(\frac{\delta_{3}\left(\tau^{-1}\left(g_{1}(s)\right), t_{1}\right)}{\delta_{3}\left(s, t_{1}\right)}\right)^{\alpha_{3}}-\frac{\rho_{+}^{\prime}(s)}{\left(\delta_{3}\left(s, t_{1}\right)\right)^{\alpha_{3}}}\right] d s=\infty,
$$

for $t_{1}$ and $t_{*}$ with $t_{*} \geq t_{1} \geq t_{0}$, then we get the same conclusion as in Theorem 3.1. 
Proof Suppose that (2.3) holds for $t \geq t_{1} \geq t_{0}$. Then $z(t)$ satisfies the two properties.

We first consider property (I). From (2.13) and (2.14), we have

$$
\begin{aligned}
r_{1}(t) z^{\alpha_{1}}(t) & \geq\left(\frac{\delta_{3}\left(t, t_{1}\right)}{\delta_{2}\left(t, t_{1}\right)}\right)^{\alpha_{1}} r_{1}(t)\left(z^{\prime}(t)\right)^{\alpha_{1}} \\
& \geq\left(\frac{\delta_{3}\left(t, t_{1}\right)}{\delta_{2}\left(t, t_{1}\right)}\right)^{\alpha_{1}} \delta_{1}\left(t, t_{1}\right) r_{2}^{\frac{1}{\alpha_{2}}}(t)\left(r_{1}(t)\left(z^{\prime}(t)\right)^{\alpha_{1}}\right)^{\prime},
\end{aligned}
$$

and

$$
z^{\alpha_{3}}(t) \geq\left(\delta_{3}\left(t, t_{1}\right)\right)^{\alpha_{3}} r_{2}(t)\left(\left(r_{1}(t)\left(z^{\prime}(t)\right)^{\alpha_{1}}\right)^{\prime}\right)^{\alpha_{2}}
$$

Define $\omega(t)$ by (3.2). As in the proof of Theorem 3.1, we get (3.3). Since $E_{2}(t)>0$, (3.3) indicates

$$
\omega^{\prime}(t) \leq \rho_{+}^{\prime}(t) \frac{r_{2}(t)\left(\left(r_{1}(t)\left(z^{\prime}(t)\right)^{\alpha_{1}}\right)^{\prime}\right)^{\alpha_{2}}}{z^{\alpha_{3}}(t)}+\rho(t) \frac{\left[r_{2}(t)\left(\left(r_{1}(t)\left(z^{\prime}(t)\right)^{\alpha_{1}}\right)^{\prime}\right)^{\alpha_{2}}\right]^{\prime}}{z^{\alpha_{3}}(t)}
$$

Combining the latter inequality with (3.5), (3.6), and (3.15), we see that

$$
\omega^{\prime}(t) \leq \frac{\rho_{+}^{\prime}(t)}{\left(\delta_{3}\left(t, t_{1}\right)\right)^{\alpha_{3}}}-\rho(t) q_{2}(t)\left(\frac{\delta_{3}\left(\tau^{-1}\left(g_{1}(t)\right), t_{1}\right)}{\delta_{3}\left(t, t_{1}\right)}\right)^{\alpha_{3}} .
$$

An integration of (3.16) from $t_{2}\left(t_{2} \geq t_{1}\right)$ to $t$ leads to

$$
\int_{t_{2}}^{t}\left[\rho(s) q_{2}(s)\left(\frac{\delta_{3}\left(\tau^{-1}\left(g_{1}(s)\right), t_{1}\right)}{\delta_{3}\left(s, t_{1}\right)}\right)^{\alpha_{3}}-\frac{\rho_{+}^{\prime}(s)}{\left(\delta_{3}\left(s, t_{1}\right)\right)^{\alpha_{3}}}\right] d s \leq \omega\left(t_{2}\right)-\omega(t)<\omega\left(t_{2}\right),
$$

for all sufficiently large $t$, which contradicts (3.14).

Secondly, if property (II) holds, then $\lim _{t \rightarrow \infty} x(t)=0$.

Theorem 3.3 Assume that (A1)-(A5), (2.1), (2.2), and (2.6) hold. Furthermore, suppose that $g_{1}(t) \leq \tau(t)$ and $\alpha_{1} \alpha_{2}=\alpha_{3} \geq 1$. If there exists $\rho(t)$ s.t.

$$
\begin{gathered}
\limsup _{t \rightarrow \infty} \int_{t_{*}}^{t}\left[\rho(s) q_{2}(s)\left(\frac{\delta_{3}\left(\tau^{-1}\left(g_{1}(s)\right), t_{1}\right)}{\delta_{3}\left(s, t_{1}\right)}\right)^{\alpha_{3}}\right. \\
\left.-\frac{\left(\rho_{+}^{\prime}(s)\right)^{2}}{4 \alpha_{3} \rho(s) \gamma(s) \delta_{2}\left(s, t_{1}\right)\left(\delta_{3}\left(s, t_{1}\right)\right)^{\alpha_{3}-1}}\right] d s=\infty,
\end{gathered}
$$

for $t_{1}$ and $t_{*}$ with $t_{*} \geq t_{1} \geq t_{0}$, where $\gamma(t)$ is given in Theorem 3.1, then we get the same conclusion as in Theorem 3.1 .

Proof Suppose that (2.3) holds for $t \geq t_{1} \geq t_{0}$. Then $z(t)$ satisfies the two properties.

We first consider property (I). Proceeding as in the proof of Theorem 3.1, we get (3.11), and

$$
\begin{gathered}
\omega^{\prime}(t) \leq \frac{\rho_{+}^{\prime}(t)}{\rho(t)} \omega(t)-\rho(t) q_{2}(t)\left(\frac{\delta_{3}\left(\tau^{-1}\left(g_{1}(t)\right), t_{1}\right)}{\delta_{3}\left(t, t_{1}\right)}\right)^{\alpha_{3}} \\
-\frac{\alpha_{3} \gamma(t) \delta_{2}\left(t, t_{1}\right) \omega^{2}(t)}{\rho^{\frac{1}{\alpha_{1} \alpha_{2}}}(t)} \omega^{\frac{1}{\alpha_{1} \alpha_{2}}-1}(t) .
\end{gathered}
$$


From (3.2) and (3.15), one has

$$
\begin{aligned}
\omega^{\frac{1}{\alpha_{1} \alpha_{2}}-1}(t) & =\rho^{\frac{1}{\alpha_{1} \alpha_{2}}-1}(t)\left(\frac{r_{2}(t)\left(\left(r_{1}(t)\left(z^{\prime}(t)\right)^{\alpha_{1}}\right)^{\prime}\right)^{\alpha_{2}}}{z^{\alpha_{3}}(t)}\right)^{\frac{1}{\alpha_{1} \alpha_{2}}-1} \\
& \geq \rho^{\frac{1}{\alpha_{1} \alpha_{2}}-1}(t)\left(\delta_{3}\left(t, t_{1}\right)\right)^{\alpha_{3}-1}
\end{aligned}
$$

We substitute (3.19) into (3.18) to see that

$$
\begin{aligned}
\omega^{\prime}(t) \leq & \frac{\rho_{+}^{\prime}(t)}{\rho(t)} \omega(t)-\rho(t) q_{2}(t)\left(\frac{\delta_{3}\left(\tau^{-1}\left(g_{1}(t)\right), t_{1}\right)}{\delta_{3}\left(t, t_{1}\right)}\right)^{\alpha_{3}} \\
& -\frac{\alpha_{3} \gamma(t) \delta_{2}\left(t, t_{1}\right)\left(\delta_{3}\left(t, t_{1}\right)\right)^{\alpha_{3}-1}}{\rho(t)} \omega^{2}(t),
\end{aligned}
$$

from which one gets

$$
\omega^{\prime}(t) \leq-\rho(t) q_{2}(t)\left(\frac{\delta_{3}\left(\tau^{-1}\left(g_{1}(t)\right), t_{1}\right)}{\delta_{3}\left(t, t_{1}\right)}\right)^{\alpha_{3}}+\frac{\left(\rho_{+}^{\prime}(t)\right)^{2}}{4 \alpha_{3} \rho(t) \gamma(t) \delta_{2}\left(t, t_{1}\right)\left(\delta_{3}\left(t, t_{1}\right)\right)^{\alpha_{3}-1}},
$$

by completing the square with respect to $\omega(t)$. We integrate the latter inequality from $t_{2}$ $\left(t_{2} \geq t_{1}\right)$ to $t$ to obtain

$$
\begin{aligned}
\int_{t_{2}}^{t} & {\left[\rho(s) q_{2}(s)\left(\frac{\delta_{3}\left(\tau^{-1}\left(g_{1}(s)\right), t_{1}\right)}{\delta_{3}\left(s, t_{1}\right)}\right)^{\alpha_{3}}\right.} \\
& \left.-\frac{\left(\rho_{+}^{\prime}(s)\right)^{2}}{4 \alpha_{3} \rho(s) \gamma(s) \delta_{2}\left(s, t_{1}\right)\left(\delta_{3}\left(s, t_{1}\right)\right)^{\alpha_{3}-1}}\right] d s \leq \omega\left(t_{2}\right),
\end{aligned}
$$

for all sufficiently large $t$, which contradicts (3.17).

Secondly, if property (II) holds, then $\lim _{t \rightarrow \infty} x(t)=0$.

Next, we consider $g_{1}(t) \geq \tau(t)$ for $t \geq t_{0}$.

Theorem 3.4 Assume that conditions (A1)-(A5), (2.1), (2.2), and (2.6) hold, and $g_{1}(t) \geq$ $\tau(t)$. If there exists $\rho(t)$ s.t.

$$
\limsup _{t \rightarrow \infty} \int_{t_{*}}^{t}\left[\rho(s) q_{2}(s)-\frac{\lambda\left(\rho_{+}^{\prime}(s)\right)^{\alpha_{1} \alpha_{2}+1}}{\left(\rho(s) \gamma(\tau(s)) \delta_{2}\left(\tau(s), t_{1}\right)\right)^{\alpha_{1} \alpha_{2}}}\right] d s=\infty,
$$

for $t_{1}$ and $t_{*}$ with $t_{*} \geq t_{1} \geq t_{0}$, then we get the same conclusion as in Theorem 3.1.

Proof Suppose that (2.3) holds for $t \geq t_{1} \geq t_{0}$. Then $z(t)$ satisfies the two properties.

We first consider property (I). Define $v(t)$ by

$$
v(t)=\rho(t) \frac{r_{2}(t)\left(\left(r_{1}(t)\left(z^{\prime}(t)\right)^{\alpha_{1}}\right)^{\prime}\right)^{\alpha_{2}}}{z^{\alpha_{3}}(\tau(t))}, \quad t \geq t_{1}
$$

Then $v(t)>0$ and

$$
\begin{aligned}
v^{\prime}(t)= & \rho^{\prime}(t) \frac{r_{2}(t)\left(\left(r_{1}(t)\left(z^{\prime}(t)\right)^{\alpha_{1}}\right)^{\prime}\right)^{\alpha_{2}}}{z^{\alpha_{3}}(\tau(t))}+\rho(t)\left[\frac{\left[r_{2}(t)\left(\left(r_{1}(t)\left(z^{\prime}(t)\right)^{\alpha_{1}}\right)^{\prime}\right)^{\alpha_{2}}\right]^{\prime}}{z^{\alpha_{3}}(\tau(t))}\right. \\
& \left.-\frac{\alpha_{3} r_{2}(t)\left(\left(r_{1}(t)\left(z^{\prime}(t)\right)^{\alpha_{1}}\right)^{\prime}\right)^{\alpha_{2}}(z(\tau(t)))^{\prime}}{z^{\alpha_{3}+1}(\tau(t))}\right]
\end{aligned}
$$




$$
\begin{aligned}
= & \frac{\rho^{\prime}(t)}{\rho(t)} v(t)+\rho(t) \frac{\left[r_{2}(t)\left(\left(r_{1}(t)\left(z^{\prime}(t)\right)^{\alpha_{1}}\right)^{\prime}\right)^{\alpha_{2}}\right]^{\prime}}{z^{\alpha_{3}}(\tau(t))} \\
& -\alpha_{3} \rho(t) \frac{r_{2}(t)\left(\left(r_{1}(t)\left(z^{\prime}(t)\right)^{\alpha_{1}}\right)^{\prime}\right)^{\alpha_{2}}(z(\tau(t)))^{\prime}}{z^{\alpha_{3}+1}(\tau(t))} .
\end{aligned}
$$

Since $\tau^{-1}\left(g_{1}(t)\right) \geq t \geq \tau(t)$ and $z^{\prime}(t)>0$, we have

$$
\frac{z\left(\tau^{-1}\left(g_{1}(t)\right)\right)}{z(\tau(t))} \geq 1, \quad t \geq t_{1},
$$

which indicates that

$$
\frac{\left[r_{2}(t)\left(\left(r_{1}(t)\left(z^{\prime}(t)\right)^{\alpha_{1}}\right)^{\prime}\right)^{\alpha_{2}}\right]^{\prime}}{z^{\alpha_{3}}(\tau(t))} \leq-q_{2}(t)
$$

due to (2.12). Based on (3.7), $E_{2}^{\prime}(t) \leq 0$ and $\tau(t) \leq t$, so one has $\tau(t) \geq t_{1}$ and

$$
(z(\tau(t)))^{\prime} \geq \delta_{2}\left(\tau(t), t_{1}\right)\left(r_{2}(t)\left(\left(r_{1}(t)\left(z^{\prime}(t)\right)^{\alpha_{1}}\right)^{\prime}\right)^{\alpha_{2}}\right)^{\frac{1}{\alpha_{1} \alpha_{2}}},
$$

for $t \geq t_{2}>t_{1}$. Combining (3.9), (3.10), (3.23)-(3.25), we conclude that

$$
\begin{aligned}
v^{\prime}(t) \leq & \frac{\rho_{+}^{\prime}(t)}{\rho(t)} v(t)-\rho(t) q_{2}(t) \\
& -\frac{\alpha_{3} \gamma(\tau(t)) \delta_{2}\left(\tau(t), t_{1}\right)}{\rho^{\frac{1}{\alpha_{1} \alpha_{2}}}(t)} v^{\frac{1}{\alpha_{1} \alpha_{2}}+1}(t) .
\end{aligned}
$$

Using (3.12) and (3.26) with

$$
B=\frac{\rho_{+}^{\prime}(t)}{\rho(t)}, \quad A=\frac{\alpha_{3} \gamma(\tau(t)) \delta_{2}\left(\tau(t), t_{1}\right)}{\rho^{\frac{1}{\alpha_{1} \alpha_{2}}}(t)},
$$

one gets

$$
v^{\prime}(t) \leq-\rho(t) q_{2}(t)+\frac{\lambda\left(\rho_{+}^{\prime}(t)\right)^{\alpha_{1} \alpha_{2}+1}}{\left(\rho(t) \gamma(\tau(t)) \delta_{2}\left(\tau(t), t_{1}\right)\right)^{\alpha_{1} \alpha_{2}}} .
$$

Integrating the latter inequality from $t_{2}$ to $t$, we have

$$
\int_{t_{2}}^{t}\left[\rho(s) q_{2}(s)-\frac{\lambda\left(\rho_{+}^{\prime}(s)\right)^{\alpha_{1} \alpha_{2}+1}}{\left(\rho(s) \gamma(\tau(s)) \delta_{2}\left(\tau(s), t_{1}\right)\right)^{\alpha_{1} \alpha_{2}}}\right] d s \leq \omega\left(t_{2}\right),
$$

for all sufficiently large $t$, which contradicts (3.20).

Secondly, if property (II) holds, then $\lim _{t \rightarrow \infty} x(t)=0$.

Theorem 3.5 Assume that (A1)-(A5), (2.1), (2.2), and (2.6) hold. Furthermore, suppose that $g_{1}(t) \geq \tau(t)$ and $\alpha_{1} \alpha_{2}=\alpha_{3}$. If there exists $\rho(t)$ s.t.

$$
\limsup _{t \rightarrow \infty} \int_{t_{*}}^{t}\left[\rho(s) q_{2}(s)-\frac{\rho_{+}^{\prime}(s)}{\left(\delta_{3}\left(\tau(s), t_{1}\right)\right)^{\alpha_{3}}}\right] d s=\infty,
$$

for $t_{1}$ and $t_{*}$ with $t_{*} \geq t_{1} \geq t_{0}$, then we get the same conclusion as in Theorem 3.1. 
Proof Suppose that (2.3) holds for $t \geq t_{1} \geq t_{0}$. Then $z(t)$ satisfies the two properties.

We first consider property (I). Proceeding as in the proof of Theorem 3.4, we get (3.22), which implies

$$
v^{\prime}(t) \leq \rho_{+}^{\prime}(t) \frac{r_{2}(t)\left(\left(r_{1}(t)\left(z^{\prime}(t)\right)^{\alpha_{1}}\right)^{\prime}\right)^{\alpha_{2}}}{z^{\alpha_{3}}(\tau(t))}+\rho(t) \frac{\left[r_{2}(t)\left(\left(r_{1}(t)\left(z^{\prime}(t)\right)^{\alpha_{1}}\right)^{\prime}\right)^{\alpha_{2}}\right]^{\prime}}{z^{\alpha_{3}}(\tau(t))} .
$$

Applying (3.15), the monotonicity of $E_{2}(t)$ and the fact that $\tau(t) \leq t$, one has $\tau(t) \geq t_{1}$ and

$$
z^{\alpha_{3}}(\tau(t)) \geq\left(\delta_{3}\left(\tau(t), t_{1}\right)\right)^{\alpha_{3}} r_{2}(t)\left(\left(r_{1}(t)\left(z^{\prime}(t)\right)^{\alpha_{1}}\right)^{\prime}\right)^{\alpha_{2}}
$$

for $t \geq t_{2}>t_{1}$. Combining (3.24), (3.28), and (3.29), one gets

$$
v^{\prime}(t) \leq \frac{\rho_{+}^{\prime}(t)}{\left(\delta_{3}\left(\tau(t), t_{1}\right)\right)^{\alpha_{3}}}-\rho(t) q_{2}(t)
$$

Upon integrating (3.30) from $t_{2}$ to $t$, we obtain a contradiction to (3.27).

Secondly, if property (II) holds, then $\lim _{t \rightarrow \infty} x(t)=0$.

Theorem 3.6 Assume that (A1)-(A5), (2.1), (2.2), and (2.6) hold. Furthermore, suppose that $g_{1}(t) \geq \tau(t)$ and $\alpha_{1} \alpha_{2}=\alpha_{3} \geq 1$. If there exists $\rho(t)$ s.t.

$$
\limsup _{t \rightarrow \infty} \int_{t_{*}}^{t}\left[\rho(s) q_{2}(s)-\frac{\left(\rho_{+}^{\prime}(s)\right)^{2}}{4 \alpha_{3} \rho(s) \gamma(\tau(s)) \delta_{2}\left(\tau(s), t_{1}\right)\left(\delta_{3}\left(\tau(s), t_{1}\right)\right)^{\alpha_{3}-1}}\right] d s=\infty,
$$

for $t_{1}$ and $t_{*}$ with $t_{*} \geq t_{1} \geq t_{0}$, then we get the same conclusion as in Theorem 3.1.

We omit the proof of Theorem 3.6 here, since it is similar to that of Theorem 3.3.

\section{Examples}

The following examples are given to show the applications of Theorems 3.1 and 3.5.

Example 4.1 For $t>k_{1} \geq 1$, consider a TONDDE with DDA

$$
E_{2}^{\prime}(t)+\int_{k_{1}}^{k_{1}+1} 10(t+\xi)\left|x\left(t-k_{1}-\frac{1}{\xi}\right)\right|^{\frac{4}{3}} x\left(t-k_{1}-\frac{1}{\xi}\right) d \xi=0
$$

where

$$
\begin{aligned}
& E_{2}(t)=\left|E_{1}^{\prime}(t)\right|^{4} E_{1}^{\prime}(t), \\
& E_{1}(t)=\left(t-k_{1}\right)\left|z^{\prime}(t)\right|^{-\frac{2}{3}} z^{\prime}(t), \\
& z(t)=x(t)+\frac{4 t+5}{t+1} x\left(t-k_{1}\right) .
\end{aligned}
$$

Let $\alpha_{1}=1 / 3, \alpha_{2}=5, \alpha_{3}=7 / 3, a=k_{1}, b=k_{1}+1, r_{1}(t)=t-k_{1}, r_{2}(t)=1, \tau(t)=t-k_{1}, g(t, \xi)=$ $t-k_{1}-1 / \xi, \sigma(\xi)=\xi, p(t)=(4 t+5) /(t+1), q(t, \xi)=10(t+\xi)$. Choose $t_{0}=t_{1}=k_{1}$. Then we obtain $\alpha_{1} \alpha_{2}<\alpha_{3}, 4 \leq p(t)<5$,

$$
g_{1}(t)=g\left(t, k_{1}\right)=t-k_{1}-\frac{1}{k_{1}}
$$




$$
\begin{aligned}
& \delta_{1}\left(t, t_{1}\right)=\delta_{1}\left(t, k_{1}\right)=t-k_{1}, \\
& \delta_{2}\left(t, t_{1}\right)=\left(\frac{\delta_{1}\left(t, k_{1}\right)}{t-k_{1}}\right)^{3}=1, \\
& \delta_{3}\left(t, t_{1}\right)=\delta_{3}\left(t, k_{1}\right)=t-k_{1}, \\
& \delta_{3}\left(\tau^{-1}(t), t_{1}\right)=\delta_{3}\left(t+k_{1}, k_{1}\right)=t \\
& \delta_{3}\left(\tau^{-1}\left(\tau^{-1}(t)\right), t_{1}\right)=\delta_{3}\left(t+2 k_{1}, k_{1}\right)=t+k_{1}, \\
& \delta_{3}\left(\tau^{-1}\left(g_{1}(t)\right), t_{1}\right)=\delta_{3}\left(t-\frac{1}{k_{1}}, k_{1}\right)=t-k_{1}-\frac{1}{k_{1}} .
\end{aligned}
$$

Furthermore, we deduce that

$$
\begin{aligned}
& p_{1}(t)>\frac{1}{5}\left(1-\frac{1}{4}\right)=\frac{3}{20}>0, \\
& p_{2}(t)>\frac{1}{5}\left(1-\frac{1}{4} \cdot \frac{t+k_{1}}{t}\right)>\frac{1}{10}>0, \\
& q_{1}(t)>\int_{k_{1}}^{k_{1}+1} \frac{3}{20} \cdot 10(t+\xi) d \xi=\frac{3}{2}\left(t+k_{1}+\frac{1}{2}\right), \\
& q_{2}(t)>\int_{k_{1}}^{k_{1}+1} \frac{1}{10} \cdot 10(t+\xi) d \xi=t+k_{1}+\frac{1}{2} .
\end{aligned}
$$

It is easy to verify that

$$
\begin{aligned}
& \int_{t_{0}}^{\infty}\left[\frac{1}{r_{1}(u)} \int_{u}^{\infty}\left(\frac{1}{r_{2}(v)} \int_{v}^{\infty} q_{1}(s) d s\right)^{\frac{1}{\alpha_{2}}} d v\right]^{\frac{1}{\alpha_{1}}} d u \\
& \quad>\int_{k_{1}}^{\infty}\left[\frac{1}{u-k_{1}} \int_{u}^{\infty}\left(\int_{v}^{\infty} \frac{3}{2}\left(s+k_{1}+\frac{1}{2}\right) d s\right)^{\frac{1}{5}} d v\right]^{3} d u \\
& \quad=\infty
\end{aligned}
$$

Therefore, conditions (A1)-(A5), (2.1), (2.2), and (2.6) hold, and $g_{1}(t) \leq \tau(t)$. We choose $\rho(t)=t$ and $t_{*}=k_{1}+2$. Applying Theorem 3.1, it remains to check (3.1), where

$$
\lambda=\left(\frac{5}{7}\right)^{\frac{5}{3}}\left(\frac{3}{8}\right)^{\frac{8}{3}}
$$

Then we get

$$
\begin{gathered}
\int_{t_{*}}^{t}\left[\rho(s) q_{2}(s)\left(\frac{\delta_{3}\left(\tau^{-1}\left(g_{1}(s)\right), t_{1}\right)}{\delta_{3}\left(s, t_{1}\right)}\right)^{\alpha_{3}}-\frac{\lambda\left(\rho_{+}^{\prime}(s)\right)^{\alpha_{1} \alpha_{2}+1}}{\left(\rho(s) \gamma(s) \delta_{2}\left(s, t_{1}\right)\right)^{\alpha_{1} \alpha_{2}}}\right] d s \\
>\int_{k_{1}+2}^{t}\left[s\left(s+k_{1}+\frac{1}{2}\right)\left(\frac{s-k_{1}-\frac{1}{k_{1}}}{s-k_{1}}\right)^{\frac{7}{3}}-\frac{\left(\frac{5}{7}\right)^{\frac{5}{3}}\left(\frac{3}{8}\right)^{\frac{8}{3}}}{\left(m_{2} s\right)^{\frac{5}{3}}}\right] d s \rightarrow \infty,
\end{gathered}
$$

as $t \rightarrow \infty$, since $\int_{k_{1}+2}^{t} s^{-\frac{5}{3}} d s<\infty$. Hence, we get the same conclusion as in Theorem 3.1. 
Example 4.2 For $t>k_{1} \geq 1$, consider a TONDDE with DDA

$$
E_{2}^{\prime}(t)+\int_{k_{1}}^{k_{1}+l} \frac{40 \xi}{t}\left|x\left(\frac{t+\xi}{2}\right)\right|^{2} x\left(\frac{t+\xi}{2}\right) d \xi=0
$$

where $l$ is a positive integer,

$$
\begin{aligned}
& E_{2}(t)=\left|E_{1}^{\prime}(t)\right|^{-\frac{2}{3}} E_{1}^{\prime}(t), \\
& E_{1}(t)=\left(t-k_{1}\right)\left|z^{\prime}(t)\right|^{8} z^{\prime}(t), \\
& z(t)=x(t)+\frac{5 t+4 k_{1}}{t+k_{1}} x\left(\frac{t}{2}\right) .
\end{aligned}
$$

Let $\alpha_{1}=9, \alpha_{2}=1 / 3, \alpha_{3}=3, a=k_{1}, b=k_{1}+l, r_{1}(t)=t-k_{1}, r_{2}(t)=1, \sigma(\xi)=\xi$,

$$
\tau(t)=\frac{t}{2}, \quad g(t, \xi)=\frac{t+\xi}{2}, \quad p(t)=\frac{5 t+4 k_{1}}{t+k_{1}}, \quad q(t, \xi)=\frac{40 \xi}{t}
$$

Choose $t_{0}=t_{1}=k_{1}$. Then we have $\alpha_{1} \alpha_{2}=\alpha_{3}, 4<p(t)<5$,

$$
\begin{aligned}
& g_{1}(t)=g\left(t, k_{1}\right)=\frac{t+k_{1}}{2}, \\
& \delta_{3}\left(\tau^{-1}(t), t_{1}\right)=\delta_{3}\left(2 t, k_{1}\right)=2 t-k_{1}, \\
& \delta_{3}\left(\tau^{-1}\left(\tau^{-1}(t)\right), t_{1}\right)=\delta_{3}\left(4 t, k_{1}\right)=4 t-k_{1}, \\
& \delta_{3}\left(\tau(t), t_{1}\right)=\delta_{3}\left(\frac{t}{2}, k_{1}\right)=\frac{t}{2}-k_{1}>\frac{t}{4},
\end{aligned}
$$

where $t \geq t_{2}>4 k_{1}, \delta_{1}\left(t, t_{1}\right), \delta_{2}\left(t, t_{1}\right)$, and $\delta_{3}\left(t, t_{1}\right)$ are the same as in Example 4.1. Furthermore, we deduce that

$$
\begin{aligned}
& p_{1}(t)>\frac{1}{5}\left(1-\frac{1}{4}\right)=\frac{3}{20}>0 \\
& p_{2}(t)>\frac{1}{5}\left(1-\frac{1}{4} \cdot \frac{4 t-k_{1}}{2 t-k_{1}}\right)>\frac{1}{20}>0 \\
& q_{1}(t)>\int_{k_{1}}^{k_{1}+1} \frac{3}{20} \cdot \frac{40 \xi}{t} d \xi=\frac{6 k_{1} l+3 l^{2}}{t} \\
& q_{2}(t)>\int_{k_{1}}^{k_{1}+1} \frac{1}{20} \cdot \frac{40 \xi}{t} d \xi=\frac{2 k_{1} l+l^{2}}{t}
\end{aligned}
$$

Clearly, (2.6) holds. Choosing $\rho(t)=t^{2}$ and $t_{*}=t_{2}$, one has

$$
\begin{aligned}
& \int_{t_{*}}^{t}\left[\rho(s) q_{2}(s)-\frac{\rho_{+}^{\prime}(s)}{\left(\delta_{3}\left(\tau(s), t_{1}\right)\right)^{\alpha_{3}}}\right] d s \\
& \quad>\int_{t_{2}}^{t}\left[s^{2} \cdot \frac{2 k_{1} l+l^{2}}{s}-\frac{2 s}{\left(\frac{s}{4}\right)^{3}}\right] d s \rightarrow \infty,
\end{aligned}
$$

as $t \rightarrow \infty$, which means that (3.27) holds, and all conditions of Theorem 3.5 are satisfied. Hence, we get the same conclusion as in Theorem 3.1. 


\section{Funding}

This research was supported by the National Natural Science Foundation of China (Grant No. 61803176, 61703180) and A Project of Shandong Province Higher Educational Science and Technology Program (Grant No. J18KA230).

\section{Competing interests}

The authors declare that they have no competing interests.

\section{Authors' contributions}

All authors contributed equally to this work. They all read and approved the final version of the manuscript.

\section{Publisher's Note}

Springer Nature remains neutral with regard to jurisdictional claims in published maps and institutional affiliations.

Received: 15 February 2019 Accepted: 17 July 2019 Published online: 25 July 2019

\section{References}

1. Sun, Y.B., Han, Z.L., Li, T.X., Zhang, G.R.: Oscillation criteria for second-order quasilinear neutral delay dynamic equations on time scales. Adv. Differ. Equ. 2010, 1 (2010)

2. Li, T.X., Saker, S.H.: A note on oscillation criteria for second-order neutral dynamic equations on isolated time scales. Commun. Nonlinear Sci. Numer. Simul. 19(12), 4185-4188 (2014)

3. Li, T.X., Baculíková, B., Džurina, J.: Oscillatory behavior of second-order nonlinear neutral differential equations with distributed deviating arguments. Bound. Value Probl. 2014, 68, 1-15 (2014)

4. Sun, Y.B., Han, Z.L., Zhao, P., Sun, Y.: Oscillation theorems for certain second order nonlinear differential equations. J. Appl. Math. Inform. 29(5-6), 1557-1569 (2011)

5. Sun, Y.B., Han, Z.L., Sun, S.R., Zhang, C.: Interval oscillation criteria for second order nonlinear forced dynamic equations with damping on time scales. Abstr. Appl. Anal. 2013, 1 (2013)

6. Han, Z.L., Sun, Y.B., Yang, D.W., Xu, M.R.: Interval oscillation criteria for second-order damped differential equations with mixed nonlinearities. Bull. Malays. Math. Sci. Soc. 39(3), 861-884 (2016)

7. Tunç, E., Graef, J.R.: Oscillation results for second order neutral dynamic equations with distributed deviating arguments. Dyn. Syst. Appl. 23(2-3), 289-303 (2014)

8. Grace, S.R., Graef, J.R., Tunç, E.: Oscillatory behavior of second order damped neutral differential equations with distributed deviating arguments. Miskolc Math. Notes 18(2), 759-769 (2017)

9. Sun, Y.B., Han, Z.L., Sun, Y., Pan, Y.Y.: Oscillation theorems for certain third order nonlinear delay dynamic equations on time scales. Electron. J. Qual. Theory Differ. Equ. 75, 1 (2011)

10. Agarwal, R.P., Bohner, M., Li, T.X., Zhang, C.H.: Oscillation of third-order nonlinear delay differential equations. Taiwan. J. Math. 17(2), 545-558 (2013)

11. Agarwal, R.P., Bohner, M., Li, T.X., Zhang, C.H.: Hille and Nehari type criteria for third order delay dynamic equations. J. Differ. Equ. Appl. 19, 1563-1579 (2013)

12. Agarwal, R.P., Bohner, M., Li, T.X., Zhang, C.H.: A Philos-type theorem for third-order nonlinear retarded dynamic equations. Appl. Math. Comput. 249, 527-531 (2014)

13. Sun, Y.B., Han, Z.L., Sun, S.R., Zhang, C.: Oscillation criteria for even order nonlinear neutral differential equations. Electron. J. Qual. Theory Differ. Equ. 30, 1 (2012)

14. Sun, Y.B., Han, Z.L.: Oscillation of even order damped neutral delay differential equations with mixed nonlinearities. In: Proc. 2012 Inter. Confer. Ind. Control Electron. Eng. Vol. 775(3), pp. 1777-1780 (2012)

15. Li, T.X., Rogovchenko, Y.V.: Asymptotic behavior of an odd-order delay differential equation. Bound. Value Probl. 2014 $1,1-10(2014)$

16. Graef, J.R., Grace, S.R., Tunç, E.: Oscillation criteria for even-order differential equations with unbounded neutral coefficients and distributed deviating arguments. Funct. Differ. Equ. 25(3-4), 143-153 (2018)

17. Feng, Q.H.: Oscillatory and asymptotic criteria of third order nonlinear delay dynamic equations with damping term on time scales. J. Appl. Anal. Comput. 8(4), 1260-1281 (2018)

18. Baculíková, B., Džurina, J.: Oscillation of third-order neutral differential equations. Math. Comput. Model. 52(1-2), $215-226(2010)$

19. Han, Z.L., Sun, Y.B., Zhang, C.H.: Oscillation of third-order nonlinear neutral delay dynamic equations. In: Proc. 7th Confer. Biological Dyn. Syst. Stab. Differ. Equ., pp. 897-900 (2010)

20. Li, T.X., Zhang, C.H., Xing, G.J.: Oscillation of third-order neutral delay differential equations. Abstr. Appl. Anal. 2012, 1 (2012)

21. Zhang, C.H., Saker, S.H., Li, T.X.: Oscillation of third-order neutral dynamic equations on time scales. Dyn. Contin. Discrete Impuls. Syst., Ser. B, Appl. Algorithms 3(3), 333-358 (2013)

22. Jiang, Y., Li, T.X.: Asymptotic behavior of a third-order nonlinear neutral delay differential equation. J. Inequal. Appl. 2014, 2014512, 1-7 (2014)

23. Utku, N., Şenel, M.T.: Oscillation behavior of third-order quasilinear neutral delay dynamic equations on time scales. Filomat 28(7), 1425-1436 (2014)

24. Jiang, Y., Jiang, C.M., Li, T.X.: Oscillatory behavior of third-order nonlinear neutral delay differential equations. Adv. Differ. Equ. 2016, 1 (2016)

25. Sun, Y.B., Han, Z.L., Zhang, Y.X.: On the oscillation for third-order nonlinear neutral delay dynamic equations on time scales. J. Appl. Math. Comput. 54(1), 243-261 (2017)

26. Grace, S.R., Graef, J.R., Tunç, E.: Oscillatory behavior of a third-order neutral dynamic equation with distributed delays. Electron. J. Qual. Theory Differ. Equ. 14, 1 (2016)

27. Zhang, Q.X., Gao, L., Yu, Y.H.: Oscillation criteria for third-order neutral differential equations with continuously distributed delay. Appl. Math. Lett. 25(10), 1514-1519 (2012) 
28. Tian, Y.Z., Cai, Y.L., Fu, Y.L., Li, T.X.: Oscillation and asymptotic behavior of third-order neutral differential equations with distributed deviating arguments. Adv. Differ. Equ. 2015, 267, 1-14 (2015)

29. Fu, Y.L., Tian, Y.Z., Jiang, C.M., Li, T.X.: On the asymptotic properties of nonlinear third-order neutral delay differential equations with distributed deviating arguments. J. Funct. Spaces 2016, 1-5 (2016)

30. Jiang, C.M., Li, T.X.: Oscillation criteria for third-order nonlinear neutral differential equations with distributed deviating arguments. J. Nonlinear Sci. Appl. 9, 6170-6182 (2016)

31. Elabbasy, E.M., Moaaz, O.: New oscillation results for class of third order neutral delay differential equations with distributed deviating arguments. Glob. J. Sci. Front. Res. 15(9), 1-9 (2015)

32. Wang, H.X., Chen, G.J., Jiang, Y., Jiang, C.M., Li, T.X.: Asymptotic behavior of third-order neutral differential equations with distributed deviating arguments. J. Math. Comput. Sci. 17, 194-199 (2017)

33. Tunç, E.: Oscillatory and asymptotic behavior of third-order neutral differential equations with distributed deviating arguments. Electron. J. Differ. Equ. 16, 1 (2017)

34. Jiang, C.M., Jiang, Y., Li, T.X.: Asymptotic behavior of third-order differential equations with nonpositive neutral coefficients and distributed deviating arguments. Adv. Differ. Equ. 2016, 1 (2016)

35. Graef, J.R., Tunç, E., Grace, S.R.: Oscillatory and asymptotic behavior of a third-order nonlinear neutral differential equation. Opusc. Math. 37(6), 839-852 (2017)

\section{Submit your manuscript to a SpringerOpen ${ }^{\circ}$ journal and benefit from:}

- Convenient online submission

- Rigorous peer review

- Open access: articles freely available online

- High visibility within the field

- Retaining the copyright to your article 